\title{
A note on eigenvalues of random block Toeplitz matrices with slowly growing bandwidth
}

\author{
Yi-Ting Li, Dang-Zheng Liu, Xin Sun and Zheng-Dong Wang \\ School of Mathematical Sciences \\ Peking University \\ Beijing, 100871, P. R. China
}

March 1, 2011

\begin{abstract}
This paper can be thought of as a remark of [5], where the authors studied the eigenvalue distribution $\mu_{X_{N}}$ of random block Toeplitz band matrices with given block order $m$. In this note we will give explicit density functions of $\lim _{N \rightarrow \infty} \mu_{X_{N}}$ when the bandwidth grows slowly. In fact, these densities are exactly the normalized one-point correlation functions of $m \times m$ Gaussian unitary ensemble (GUE for short). The series $\left\{\lim _{N \rightarrow \infty} \mu_{X_{N}} \mid m \in \mathbb{N}\right\}$ can be seen as a transition from the standard normal distribution to semicircle distribution. We also show a similar relationship between GOE and block Toeplitz band matrices with symmetric blocks.

Keywords: block Toeplitz matrix, GUE, GOE, limit spectral distribution
\end{abstract}

\section{Introduction}

A block Toeplitz matrix is a block matrix which can be written as

$$
T_{N}=\left(A_{i-j}\right)_{i, j=1}^{N}=\left(\begin{array}{ccccc}
A_{0} & A_{-1} & A_{-2} & \cdots & A_{-(N-1)} \\
A_{1} & A_{0} & A_{-1} & \cdots & A_{-(N-2)} \\
A_{2} & A_{1} & A_{0} & \cdots & A_{-(N-3)} \\
\vdots & \vdots & \vdots & \ddots & \vdots \\
A_{N-1} & A_{N-2} & A_{N-3} & \cdots & A_{0}
\end{array}\right)
$$

where $\left\{A_{-(N-1)}, \ldots, A_{0}, \ldots, A_{N-1}\right\}$ are $m \times m$ matrices and $A_{s}=\left(a_{i j}(s)\right)_{i, j=1}^{m}$. If the $a_{i j}(s)$ 's are random variables, then we call $T_{N}$ a random block Toeplitz matrix. We suppose the $a_{i j}(s)$ 's are real random variables and $A_{s}=\left(A_{-s}\right)^{T}$. Besides, we list some assumptions as follows.

Independence of the elements:

(1) $a_{i_{1} j_{1}}\left(s_{1}\right)$ and $a_{i_{2} j_{2}}\left(s_{2}\right)$ are independent if $\left|s_{1}\right| \neq\left|s_{2}\right|$, 
(2) If $s \neq 0$ and $\left(i_{1}, j_{1}\right) \neq\left(i_{2}, j_{2}\right)$ then $a_{i_{1} j_{1}}(s)$ and $a_{i_{2} j_{2}}(s)$ are independent,

(3) If $\left(i_{1}, j_{1}\right) \neq\left(i_{2}, j_{2}\right)$ and $\left(i_{1}, j_{1}\right) \neq\left(j_{2}, i_{2}\right)$ then $a_{i_{1} j_{1}}(0)$ and $a_{i_{2} j_{2}}(0)$ are independent.

Uniform boundness condition:

(4) $\mathbb{E}\left[a_{i j}(s)\right]=0, \mathbb{E}\left[\left|a_{i j}(s)\right|^{2}\right]=1,-(N-1) \leq s \leq N-1,1 \leq i, j \leq m$

and

$$
\sup _{\substack{N \in \mathbb{N} \\-(N-1) \leq s \leq N-1}}\left\{\mathbb{E}\left[\left|a_{i j}(s)\right|^{k}\right] \mid 1 \leq i, j \leq m\right\}=C_{k, m}<+\infty
$$

and

$$
\sup _{m \in \mathbb{N}} C_{k, m}=C_{k}<+\infty .
$$

Slowly growing bandwidth condition:

(5)The block Toeplitz matrix is a band block matrix with bandwidth $b_{N}$, that is, $A_{s}=0$ for $|s|>b_{N}$. Moreover, $b_{N}$ satisfies: $\lim _{N \rightarrow \infty} b_{N}=\infty$ and $b_{N}=o(N)$.

Gaussian unitary ensemble (GUE for short) $\left(\mathcal{H}_{m}, d \mu\right)$ is the space $\mathcal{H}_{m}$ of all Hermitian $(m \times m)$-matrices with a certain Gaussian measure $d \mu$ (see [1, 6]). We will regularly use the notations $\langle\cdot\rangle_{\mathrm{GUE}}$ and $\langle\cdot\rangle_{\mathrm{TBM}}$, respectively denoting the expectations under GUE and random Toeplitz band matrices. If $H=\left(h_{i j}\right)$ is a matrix from GUE, then it is easy to see $\left\langle h_{i j} h_{j i}\right\rangle_{\mathrm{GUE}}=1$ while all other second moments are equal to zero: $\left\langle h_{i j} h_{k l}\right\rangle_{\mathrm{GUE}}=0$, whenever $(i, j) \neq(l, k)$.

If $A=\left(a_{i j}(\omega)\right)_{i, j=1}^{N}$ is a real symmetric or complex Hermitian random matrix and its entries are random variables on a probability space $\Omega$ with a probability measure $P$, then the eigenvalue distribution of $A$ is

$$
\mu_{A}=\frac{1}{N} \int_{\Omega} \sum_{j=1}^{N} \delta_{\lambda_{j}(\omega)} d P(\omega)
$$

where $\lambda_{j}(\omega)$ 's are the $N$ real eigenvalues of $A$. We have

Theorem 1.1. Let $T_{N}$ be an $m N \times m N$ random block Toeplitz matrix as above. Set $X_{N}=\frac{T_{N}}{\sqrt{2 m b_{N}}}$, then $\mu_{X_{N}}$ converges weakly to $f_{m}(x) d x$ as $N \rightarrow \infty$. Moreover, if the bandwidth satisfies $\sum_{m=1}^{\infty} b_{N}^{-2}<\infty$, then the convergence is almost sure. Here $f_{m}(x)=\frac{1}{\sqrt{m}} \sum_{j=0}^{m-1} \psi_{j}^{2}(\sqrt{m} x)$ where $\psi_{j}$ is the $j$ th nomarlized oscillator wave-function: $\psi_{j}(x)=\frac{e^{-\frac{x^{2}}{4}} H_{j}(x)}{\sqrt{\sqrt{2 \pi} j}}$ and $H_{j}$ is the jth Hermite polynomial: $H_{j}(x)=(-1)^{j} e^{\frac{x^{2}}{2}} \frac{d^{j}}{d x^{j}} e^{-\frac{x^{2}}{2}}$.

Remark. $f_{m}(x)$ is the one-point correlation function of GUE up to the scaling [6]. As $f_{1}$ is the density function of the standard normal distribution and when $m \rightarrow$ $\infty f_{m}$ converges in law to the semicircle distribution (see [5]), $\left\{\lim _{N \rightarrow \infty} \mu_{X_{N}} \mid m \in\right.$ $\mathbb{N}\}$ can be seen as a transition from $N(0,1)$ to semicircle distribution.

In [4, Kologlu, Kopp and Miller got a very similar result. They proved that the limiting eigenvalue density of symmetric block circulant Toeplitz ensemble 
is the same as the eigenvalue density of GUE (Theorem 1.4(1) of [4]). But there is an essential difference between their model and our model: In [4 the block Toeplitz matrix has a circulant structure while in this paper the block Toeplitz matrix has a band structure. In the viewpoint of combinatorics, both of the two structures are strong. So they are two different models induced from the block Toeplitz matrix model and interestingly have the same limiting eigenvalue distribution. There is also another difference: In [4] the entries of the block Toeplitz matrix are i.i.d random variables but in our model the entries only have to satisfy the independent condition.

Theorem 1.2. Let $\nu_{1}, \ldots, \nu_{r}$ be nonnegetive integers, and $\nu=\nu_{1}+\cdots+\nu_{r}$. Set $Y_{N}=\frac{T_{N}}{\sqrt{2 b_{N}}}$, then

$$
\lim _{N \rightarrow \infty} \frac{1}{N^{\nu}}\left\langle\prod_{i=1}^{r}\left(\operatorname{tr} Y_{N}^{i}\right)^{\nu_{i}}\right\rangle_{\mathrm{TBM}}=\left\langle\prod_{i=1}^{r}\left(\operatorname{tr} H^{i}\right)^{\nu_{i}}\right\rangle_{\mathrm{GUE}}
$$

\section{Proof of Main Results}

Definition 2.1. Let $[n]=\{1,2, \ldots, n\}, \forall n \in \mathbb{N}$.

(1) We call $\pi=\left\{\left\{a_{1}, b_{1}\right\}, \ldots,\left\{a_{k}, b_{k}\right\}\right\}$ a pair partition of $[2 k]$ if $\bigcup_{j=1}^{r}\left\{a_{j}, b_{j}\right\}=[2 k]$ and $\left\{a_{i}, b_{i}\right\} \bigcap\left\{a_{j}, b_{j}\right\}=\emptyset$ if $i \neq j$. For convenience, we assume that $a_{1}<\cdots<$ $a_{k}$ and $a_{i}<b_{i}$ for each pair, under which such a pair partition is called a Wick coupling. For $\pi$, we define $\pi\left(a_{j}\right)=b_{j}$ and $\pi\left(b_{j}\right)=a_{j}(1 \leq j \leq k)$. We denote by $\mathcal{P}_{2}(2 k)$ the set of pair partitions of $[2 k]$.

(2) Suppose $\pi \in \mathcal{P}_{2}(2 k)$. Then $\pi$ can be seen as a permutation: $\left(a_{1}, b_{1}\right) \cdots\left(a_{k}, b_{k}\right)$. Consider the canonical cycle $\gamma_{0}=(1,2, \ldots, 2 k-1,2 k)$. Let $g(\pi)$ denote the number of orbits of the permutation $\gamma_{0} \circ \pi$.

The following lemma is a well-known consequence of Wick's formula on the moments of GUE. One can get it with the method of moment generating function (see Section 3.3.1 of [1]).

Lemma 2.2. Suppose $H=\left(b_{i j}\right)_{i, j=1}^{m}$ is an $m \times m$ random Hermitian matrix from GUE. Set $Y=\frac{H}{\sqrt{m}}$. Then the odd moments of $\mu_{Y}$ are all 0 and the even moments of $\mu_{Y}$ are $m_{2 k}\left(\mu_{Y}\right)=\sum_{\pi \in \mathcal{P}_{2}(2 k)} m^{g(\pi)-k-1}$.

Proof of Theorem 1.1. From Theorem 4.3 of [5] and Lemma 2.2. we know that $\mu_{Y}$ and $\gamma_{T}^{m}$ have the same moments. It follows from Carleman's Theorem (see [3] that $\gamma_{T}^{m}$ should be $\mu_{Y}$. So the density function of $\gamma_{T}^{m}$ is the one-point correlation function of GUE, which is a well-known function [1, 6]:

$$
f_{m}(x)=\frac{1}{\sqrt{m}} \sum_{j=0}^{m-1} \psi_{j}^{2}(\sqrt{m} x) .
$$

From [2] we know if $\sum_{N=1}^{\infty} b_{N}^{-2}<\infty$, then the convergence is almost sure. 
Proof of Theorem 1.2. By Lemma 2.2 of [5], the main contribution in the expansion of $\frac{1}{N^{\nu}}\left\langle\prod_{i=1}^{r}\left(\operatorname{tr} Y_{N}^{i}\right)^{\nu_{i}}\right\rangle_{\mathrm{TBM}}$ comes from the pair partitions. As in the proof of Theorem 4.3 in [5], we can show that

$$
\lim _{N \rightarrow \infty} \frac{1}{N^{\nu}}\left\langle\prod_{i=1}^{r}\left(\operatorname{tr} Y_{N}^{i}\right)^{\nu_{i}}\right\rangle_{\mathrm{TBM}}= \begin{cases}0 & \text { if } \sum_{i=1}^{r} i \nu_{i} \text { is odd } \\ \sum_{\pi \in \mathcal{P}_{2}\left(\sum_{i=1}^{r} i \nu_{i}\right)} m^{F(\pi)} & \text { if } \sum_{i=1}^{r} i \nu_{i} \text { is even }\end{cases}
$$

Here the definition of $F(\pi)$ as follows: Suppose that $\sum_{i=1}^{r} i \nu_{i}=2 \tilde{\nu}$ is even and $\pi=\left\{\left\{a_{1}, b_{1}\right\}, \ldots,\left\{a_{\tilde{\nu}}, b_{\tilde{\nu}}\right\}\right\} \in \mathcal{P}_{2}(2 \tilde{\nu})$, then $F(\pi)$ denotes the number of "free indices" of the system $\left\{\begin{array}{l}t_{a_{i}}=t_{f\left(b_{1}\right)} \\ t_{b_{i}}=t_{f\left(a_{i}\right)}\end{array} \quad(1 \leq i \leq \tilde{\nu})\right.$, where $f$ is defined as below:

Set $\nu_{0}=0$. For $1 \leq x \leq \sum_{i=1}^{r} i \nu_{i}$, if $\exists 0 \leq s \leq r-1$ and $1 \leq a \leq \nu_{s+1}$ such that $x=\sum_{i=0}^{s} i \nu_{i}+(s+1) a$, then $f(x)=\sum_{i=0}^{s} i \nu_{i}+(s+1)(a-1)+1$; otherwise $f(x)=x+1$.

By Wick's formula, we can compute the integral $\left\langle\prod_{i=1}^{r}\left(\operatorname{tr} H^{i}\right)^{\nu_{i}}\right\rangle_{\mathrm{GUE}}$ and complete the proof.

\section{Similar Results for GOE and Block Toeplitz Matrix with Symmetric Blocks}

First, we remark that we can get the same results associated with GUE if one of the following conditions are imposed:

1) each block of $T_{N}$ is a complex matrix and $A_{-s}=\left(\overline{A_{s}}\right)^{T}$;

2) each block of $T_{N}$ is a Hermitian matrix, that is, $A_{-s}=A_{s}=\left(\overline{A_{s}}\right)^{T}$.

However, the situation becomes different when each block of $T_{N}=\left(A_{i-j}\right)_{i, j=1}^{N}$ is a symmetric matrix, that is, $A_{-s}=A_{s}=\left(A_{s}\right)^{T}$. Besides, we modify the second moments of each block as follows: $\mathbb{E}\left[\left|a_{i j}(s)\right|^{2}\right]=\left\{\begin{array}{ll}1 & \text { if } i \neq j \\ 2 & \text { if } i=j\end{array}, \forall s\right.$.

We still use the notations $\langle\cdot\rangle_{\mathrm{GOE}}$ and $\langle\cdot\rangle_{\mathrm{TBM}}$, respectively denoting the expectations under GOE and Toeplitz band matrices with symmetric blocks.

Theorem 3.1. Let $T_{N}$ be an $m N \times m N$ random block Toeplitz matrix as mentioned above. Set $X_{N}=\frac{T_{N}}{\sqrt{2 m b_{N}}}$, then

$$
\lim _{N \rightarrow \infty} \frac{1}{m N}\left\langle\operatorname{tr} X_{N}^{k}\right\rangle_{\mathrm{TBM}}=\frac{1}{m}\left\langle\operatorname{tr}(H / \sqrt{m})^{k}\right\rangle_{\mathrm{GOE}} .
$$


Moreover, $\mu_{X_{N}}$ converges weakly to $g_{m}(x) d x$ as $N \rightarrow \infty$. And if the bandwidth satisfies $\sum_{N=1}^{\infty} b_{N}^{-2}<\infty$, then the convergence is almost sure. Here

$g_{m}(x)=\frac{1}{\sqrt{m}} \sum_{j=0}^{m-1} \psi_{j}^{2}(\sqrt{m} x)+\left(\frac{m}{2}\right)^{1 / 2} \psi_{m-1}(\sqrt{m} x) \int_{-\infty}^{\infty} \varepsilon(x-t) \psi_{m}(\sqrt{m} t) d t+\alpha_{m}(x)$,

$\varepsilon(x)=\frac{1}{2} \operatorname{sign}(x)$ and $\psi_{j}$ is the $j$ th nomarlized oscillator wave-function as in Theorem 1.1, while

$$
\alpha_{m}(x)=\left\{\begin{array}{ll}
\frac{1}{m} \psi_{2 s}(\sqrt{m} x) \div \int_{-\infty}^{\infty} \psi_{2 s}(\sqrt{m} t) d t & \text { if } m=2 s+1 \\
0 & \text { if } m=2 s
\end{array} .\right.
$$

Proof of Theorem 3.1. For $H=\left(h_{i j}\right)_{i, j=1}^{m}$, let $Y=\frac{H}{\sqrt{m}}$. From Wick's formula we know $\frac{1}{m}\left\langle\operatorname{tr}(H / \sqrt{m})^{k}\right\rangle_{\mathrm{GOE}}=0$ when $k$ is odd and $\frac{1}{m}\left\langle\operatorname{tr}(H / \sqrt{m})^{2 k}\right\rangle_{\mathrm{GOE}}$ is

$$
m^{-k-1} \sum_{t_{1}, \ldots, t_{2 k}=1}^{m} \sum\left(\left\langle h_{t_{a_{1}} t_{a_{1}+1}} h_{t_{b_{1}} t_{b_{1}+1}}\right\rangle_{\mathrm{GOE}} \cdots\left\langle h_{t_{a_{k}} t_{a_{k}+1}} h_{t_{b_{k}} t_{b_{k}+1}}\right\rangle_{\mathrm{GOE}}\right)
$$

where the second sum is taken over all $\pi=\left\{\left\{a_{1}, b_{1}\right\}, \ldots,\left\{a_{k}, b_{k}\right\}\right\} \in \mathcal{P}_{2}(2 k)$ and $t_{2 k+1}:=t_{1} . H$ is symmetric, so $\left\langle h_{t_{a_{1}} t_{a_{1}+1}} h_{t_{b_{1}} t_{b_{1}+1}}\right\rangle_{\mathrm{GOE}} \cdots\left\langle h_{t_{a_{k}} t_{a_{k}+1}} h_{t_{b_{k}} t_{b_{k}+1}}\right\rangle_{\mathrm{GOE}} \neq$ 0 implies $\left\{\begin{array}{l}t_{a_{i}}=t_{b_{i}+1} \\ t_{b_{i}}=t_{a_{i}+1}\end{array}\right.$ or $\left\{\begin{array}{l}t_{a_{i}}=t_{b_{i}} \\ t_{a_{i}+1}=t_{b_{i}+1}\end{array} \quad\right.$ for $1 \leq i \leq k$, moreover, each term

$$
\left\langle h_{t_{a_{i}} t_{a_{i}+1}} h_{t_{b_{i}} t_{b_{i}+1}}\right\rangle_{\mathrm{GUE}}= \begin{cases}2 & \text { if } t_{a_{i}}=t_{b_{i}}=t_{a_{i}+1}=t_{b_{i}+1} \\ 1 & \text { otherwise }\end{cases}
$$

For a given $\pi=\left\{\left\{a_{1}, b_{1}\right\}, \ldots,\left\{a_{k}, b_{k}\right\}\right\} \in \mathcal{P}_{2}(2 k)$, set

$$
A(\pi)=\left\{\left(t_{1}, \ldots, t_{2 k}\right) \in[m]^{2 k} \mid\left\{\begin{array}{l}
t_{a_{i}}=t_{b_{i}+1} \\
t_{b_{i}}=t_{a_{i}+1}
\end{array} \quad \text { or }\left\{\begin{array}{l}
t_{a_{i}}=t_{b_{i}} \\
t_{a_{i}+1}=t_{b_{i}+1}
\end{array} \quad \text { for } 1 \leq i \leq k\right\} .\right.\right.
$$

For $\mathbf{t}=\left(t_{1}, \ldots, t_{2 k}\right) \in A(\pi)$, let $r(\pi, \mathbf{t})=\sharp\left\{i \in[k] \mid t_{a_{i}}=t_{b_{i}}=t_{a_{i}+1}=t_{b_{i}+1}\right\}$, then $\frac{1}{m}\left\langle\operatorname{tr}(H / \sqrt{m})^{2 k}\right\rangle_{\mathrm{GOE}}=m^{-k-1} \sum_{\pi \in \mathcal{P}_{2}(2 k)} \sum_{\mathbf{t} \in A(\pi)} 2^{r(\pi, \mathbf{t})}$.

Similarly as in the proof of Theorem 3.2 and Theorem 4.3 of [5], we know when $k$ is odd $\frac{1}{m N}\left\langle\operatorname{tr} X_{N}^{k}\right\rangle_{\mathrm{TBM}}=o(1)$ and $\frac{1}{m N}\left\langle\operatorname{tr} X_{N}^{2 k}\right\rangle_{\mathrm{TBM}}$ is

$$
\begin{aligned}
& \sum_{i=1}^{N} \sum_{j_{1}, \ldots, j_{2 k}=-b_{N}}^{b_{N}, \ldots, t_{2 k}=1} \sum_{t_{1}}^{m} \frac{E\left(a_{t_{1} t_{2}}\left(j_{1}\right) \cdots a_{t_{2 k} t_{1}}\left(j_{2 k}\right)\right)}{\left(2 m b_{N}\right)^{k} \cdot m N} \prod_{l=1}^{2 k} I_{[1, N]}\left(i+\sum_{q=1}^{l} j_{q}\right) \delta_{0, \sum_{q=1}^{2 k} j_{q}} \\
= & \sum_{i=1}^{N} \sum_{\pi \in \mathcal{P}_{2}(2 k)} \sum_{\mathbf{t} \in A(\pi)} \frac{2^{r(\pi, \mathbf{t})}}{\left(2 m b_{N}\right)^{k} \cdot m N} \sum_{x_{1}, \ldots, x_{k}=-b_{N}}^{b_{N}} \prod_{l=1}^{2 k} I_{[1, N]}\left(i+\sum_{q=1}^{l} \epsilon_{\pi}(q) x_{\pi(q)}\right)+o(1) \\
\rightarrow & \sum_{\pi \in \mathcal{P}_{2}(2 k)} \sum_{\mathbf{t} \in A(\pi)} \frac{2^{r(\pi, \mathbf{t})}}{m^{k+1}}(N \rightarrow \infty) .
\end{aligned}
$$


Thus $\lim _{N \rightarrow \infty} \frac{1}{m N}\left\langle\operatorname{tr} X_{N}^{k}\right\rangle_{\mathrm{TBM}}=\frac{1}{m}\left\langle\operatorname{tr}(H / \sqrt{m})^{k}\right\rangle_{\mathrm{GOE}}$. From [2] we know the convergence is almost sure if $\sum_{N=1}^{\infty} b_{N}^{-2}<\infty$. Finally, $g_{m}(x)$ is the one-point correlation function of GOE with order $m$, thus we complete the proof.

Remark. The density (3.3) follows from the 1-point correlation function of GOE (see (7.2.32) of [6]). This family densities can also be seen as a transition from the normal distribution $N(0,2)$ to the semicircle distribution with variance 1 . In the situation of GOE, we also have parallel results to Theorem 1.2 .

\section{Acknowledgements}

The authors thank the anonymous referee for telling us that [4 has a similar result to Theorem 1.1 .

\section{References}

[1] G. W. Anderson, A. Guionnet and O. Zeitouni An Introduction to Random Matrix, Cambridge University Press, New York, Cambridge, 2009.

[2] A. Basak and A. Bose, Limiting spectral distribution of some band matrices, Periodica Mathematica Hungarica, to appear, http://www.isical.ac.in/ statmath/html/publication/techreport.html

[3] R. Durrett, Probability: Theory and Examples, Cambridge University Press, New York, Cambridge, 2004.

[4] M. Kologlu, G. S. Kopp, S. J. Miller. The Limiting Spectral Measure for Ensembles of Symmetric Block Circulant Matrices. Available online as arXiv:1008.4812 from http://arxiv.org/abs/1008.4812

[5] Y. T. Li, D. Z. Liu and Z. D. Wang, Limit Distributions of Eigenvalues for Random Block Toeplitz and Hankel Matrices, J. Theor. Probab. (2010). doi:10.1007/s10959-010-0326-3 (http://www.springerlink.com/content/5316328u2116j242/)

[6] M. L. Mehta, Random Matrices, 3rd ed., Academic Press, San Diego, 2004.

[7] R.C. Penner, Perturbative series and the moduli space of Riemann surfaces, J. Diff. Geometry 27 (1988), 35-53.

[8] A. Zvonkin, Matrix Integrals and Map Enumeration: An Accessible Introduction, Math. Comput. Modelling 26 (1997), 281-304. 